\title{
Binge drinking and psychoactive drug use in a cohort of European youths
}

\author{
Roberta Siliquini, ${ }^{1}$ Alessandra Colombo, ${ }^{2}$ Paola Berchialla, ${ }^{1}$ Fabrizio Bert, ${ }^{2}$ TEN-D Group \\ ${ }^{1}$ Department of Public Health, University of Turin; ${ }^{2}$ School of Public Health, University of Turin, \\ Italy
}

\begin{abstract}
Significance for public health
We investigated the patterns of illegal drug and alcohol consumption in young people, focusing in particular on binge drinking behaviours, a known important current public health issue. This phenomenon has been investigated in other studies, especially those focused on the US, but to date, to our knowledge, no European studies have been conducted in the natural setting of a large cohort of young people. The TEN-D survey confirmed that the prevalence of binge drinking is higher among younger people (20-24 years old) and may be related to socio-economic variables (such as living with friend and colleagues or lower educational level). We found a significant association between the consumption of illegal drugs and binge drinking (poly-drug consumption). These findings should be considered when addressing specific prevention campaigns that focus on the groups with the highest likelihood of unhealthy behaviours.
\end{abstract}

\section{Abstract}

Background. TEN-D by Night is an international, multicentre, crosssectional portal survey conducted on a large sample of young people in six European countries. This paper aims to investigate the alcohol and psychoactive drug consumption of this sample, with a focus on the prevalence of binge drinking and the poly-drug habits of the TEN-D cohort.

Design and Methods. The study population consisted of 4695 young people attending recreational sites on weekend nights. The intervention included two questionnaires and two psychoactive substance detection tests performed at the entry and exit of the recreational sites. A multivariate logistic regression model was used to predict the probability of binge drinking.

Results. Binge drinking was reported by $20 \%$ of the males and $13 \%$ of the females $(\mathrm{P}=0.001)$ before entry into the recreational sites and by $18 \%$ of the males and $11 \%$ of the females before entry into the clubs $(\mathrm{P}<0.001)$. Poly-drug use was reported by $71 \%$ of the males and $66 \%$ of the females. Living with a parent (OR 1.57; $\mathrm{P}=0.01)$, seeking employment (OR 1.66; $\mathrm{P}=0.005)$ and cannabis consumption (several times per month and several times per week, OR 1.94 and 3.66, respectively, $\mathrm{P}<0.05)$ were associated with binge drinking.

Conclusions. Our survey showed that it is possible to identify individuals and groups at higher risk of binge drinking. This identification would allow for a focus on specific targets and would facilitate the redesign of prevention programmes. The increased use of psychoactive substances among youths should be studied extensively to promote successful prevention campaigns.

\section{Introduction}

During the last two decades, an increase has been reported in the consumption of psychoactive substances (alcohol and illegal and medical drugs) among young people in Europe. A common theme of many studies is the dynamic nature of problematic alcohol and illegal drug use. ${ }^{1-7}$ Currently, the members of the European Community show a growing interest in these issues and demonstrate a desire and a shared determination to improve their understanding of this complex social issue. ${ }^{5,8,9}$ The attention given to this topic is also based on evidence that the consumption of psychoactive substances is one of the major risk factors for road crash accidents, the leading cause of death among people younger than 30 years old. ${ }^{2,8,10}$ As reported in a previous study, almost 73,500 young people aged 16-24 died in road crash accidents in 14 European countries during the decade of $1995-2004 .{ }^{11} \mathrm{Road}$ crash accidents occur more frequently on weekend nights, ${ }^{12}$ when youths are more likely to consume large quantities of alcohol, a phenomenon known as binge drinking (defined as $\geq 5$ drinks on one occasion for men and $\geq 4$ drinks on one occasion for women). ${ }^{13-19}$ Knowledge of these patterns of psychoactive substance consumption among young people is therefore crucial for planning effective prevention programmes. In the past, several studies have examined the prevalence of illegal drugs use, especially in the US, $9,18,19$ whereas other studies have reported on specific drug use in small samples of young people in regional settings. ${ }^{20,21}$ To our knowledge, however, no study has investigated binge drinking and poly-drug use in large samples directly recruited in a natural setting, such as the recreational sites where youths spend their weekend nights (in our case, clubs and discotheques). The TEN-D (Dark, Dance, Disco, Dose, Drugs, Drive, Danger, Damage, Disability, Death) by Night project is an international, multicentre, cross-sectional survey conducted in six European countries. This study was designed to investigate psychoactive substance consumption (alcohol, cocaine, methamphetamine/MDMA, THC, amphetamine, opiates, benzodiazepines) and the relationship between this risk behaviour and driving performance in a large sample of young drivers, who were enrolled in this study at typical locations of consumption. ${ }^{22}$ Using data provided by the TEN-D survey, this study aims to examine the patterns of alcohol and illegal drug use among European youths, focusing on the prevalence of binge drinking and poly-drug use.

\section{Materials and Methods}

Details about the methodology of the study protocol have been described in a previous paper.22 Briefly, TEN-D by Night is an international, multicentre, cross-sectional portal survey funded by the European Commission. 


\section{Participants}

The required sample size was calculated using the Schlesselmann formula ${ }^{23}$ for known population sizes, assuming a two-sided alpha $=0.05$, a statistical power $=90 \%$, losses or missing $=25 \%$ and taking into account the need to stratify for each country and adjust for several potential confounders in the multivariate analysis. Based on these parameters, the overall sample consisted of 4695 young drivers who were enrolled in the study on weekend nights at recreational meeting places in six European countries [Italy (18\%), Spain (21\%), Belgium/Netherlands (19\%), Poland (21\%) and Bulgaria (21\%)]. In each country, a map of the recreational meeting places was produced based on official regional lists, and an opportunistic selection was made according to the willingness of the clubs' owners.

\section{The TEN-D by Night intervention}

In each country, the interventions were conducted by survey staff composed of at least six operators who had been specifically trained. The staff included a survey manager (responsible for supervising all field activities), one technician for a driving simulation test, at least two interviewers (responsible for the administration of questionnaires and two psychoactive substance detection tests) and two hostesses for approaching the young people.

The scheduled activities were conducted at both the entry and exit of the recreational sites.

At the entry, after the voluntary recruitment of the participants based on eligibility criteria (possession of a driver's license and age between 16 and 35 years old), the staff explained the study's protocol, collected informed consent, and assigned a personal, anonymous code to each subject.

Each participant was asked to complete a structured questionnaire and a test to assess his/her BAC (breath alcohol concentration, a proxy for blood alcohol concentration $)^{24}$ and to participate in a driving simulation (using SimuNomad3 Ecrans). ${ }^{25}$ At the exit, a second questionnaire and a saliva test 26 for several illegal drugs were administered to the participants in addition to the same BAC test and the same driving simulation performed at entry. The TEN-D by Night intervention ${ }^{22}$ is based on the portal survey technique12,15 and was applied at selected youth recreational locations on weekend nights by survey staff.

\section{The questionnaires}

Two anonymous questionnaires were developed based on previously validated questionnaires. ${ }^{3}$ The questionnaires were tested for comprehensiveness and translated into the official language of each participating country.

A total of 27 closed- and open-ended questions investigated the following topics:

- Demographic and socio-economic status (gender, age, education, occupational status, living situation);

- Medical drug consumption in the last seven days;

- Driving habits (licensing age, driving frequency);

- Alcohol and illegal drug consumption;

- Driving under the influence of psychoactive substances (self-reported driving after heavy drinking or illegal drug use or having had penalties, car crashes, or license revocation when drunk or doped in the last year);

- Alcohol consumption during the event (number of shots, glasses or bottles consumed for each different alcohol type);

- Illegal drug consumption during the event (each substance);

- Binge drinking during the event;

- Intent to drive upon exit;

- Opinions about the intervention.

\section{Statistical analysis}

The results of the analyses on the predictors of reaction time were published previously, ${ }^{27}$ including descriptive statistics for males and females and the categorical variables based on relative frequencies. Differences were compared using the chi-squared test. A multivariate logistic regression model was fitted through a backward stepwise elimination analysis to predict the probability of binge drinking. The bestfitting model was chosen based on the Akaike information criterion. Finally, the goodness of fit was evaluated using the Brier score, which quantifies the overall accuracy of the predictions (for sensible models, the Brier score ranges from 0 , perfect, to 0.25 , worthless), and the $c$ index (the closer to the absolute value of one, the better), which represents the concordance between the predicted and observed responses. In the absence of an external data source, all goodness-of-fit indices were computed using a bootstrap method to account for the degree of optimism in the model accuracy evaluations induced by the use of the same data source for training and testing purposes. ${ }^{28} \mathrm{~A}$ two-tailed $\mathrm{p}$ value of 0.05 was considered significant for all analyses, which were conducted using the R statistical package version 2.9. ${ }^{29}$

Finally, based on the multivariate logistic model, a nomogram was produced to estimate the likelihood of engaging in binge drinking behaviour for each subject with specific socio-demographic characteristics. Nomograms are graphs that allow for the approximate calculation of output from mathematical functions. ${ }^{30}$

To compute the probability of engaging in binge drinking, each covariate in our nomogram is represented by a horizontal line, with tick marks indicating the categories of an explanatory variable. Given a risk profile, that is, a set of covariates (age, living situation, education and marijuana and cocaine consumption), the likelihood is derived as follows. A vertical line is drawn from each covariate's category to the Points axis at the top of the nomogram. The corresponding value is the partial risk score relative to each predictor. The sum of all of the partial risk scores is reported on the Total Points axis, and the corresponding probability is obtained by drawing a vertical line to the lower Predicted Value.

\section{Results}

\section{Description of the sample}

Overall, 4695 subjects participated in the survey. Of the participants, $67.9 \%$ were male, and the most represented age class was the 20 - to 24 year-olds.More than $80 \%$ of the subjects had at least a high school education, and the majority of the participants lived with their parents. The characteristics of the sample, according to the socio-demographic variables and psychoactive substance consumption, are reported in Table 1.

\section{Alcohol and illegal drug habits}

Regular alcohol drinking (alcohol use in the last month) was investigated. Only $7.0 \%$ of the males and $10.5 \%$ of the females reported no alcohol consumption, whereas approximately $40 \%$ of both males and females reported drinking once per week and $36.8 \%$ of the males and $19.1 \%$ of the females reported drinking regularly several times per week. Daily consumption was reported by $11.6 \%$ of the males and $3.3 \%$ of the females. Approximately half of the participants, both males and females, reported being drunk at least once in the past month $(56.5 \%$ and $45.0 \%$, respectively).

Routine illegal drug use was also evaluated (illegal drugs consumed in the last year). Most of the participants denied illegal drug consumption ( $63.9 \%$ of the males, $69.7 \%$ of the females). Among the illegal drug users, the most frequent pattern was occasional $(15.3 \%$ of the males, $15.7 \%$ of the females), followed by multi-week ( $9.7 \%$ and $6.4 \%$, respec- 
tively). Table 2 shows the distribution of specific illegal drugs consumed in the last year by gender.

The most widespread illegal drugs were marijuana and cocaine. Overall, $31.9 \%$ of the males and $26.9 \%$ of the females reported marijuana consumption in the last year, whereas $10.1 \%$ of the males and $5.8 \%$ of the females reported cocaine consumption. In the case of other psychoactive drugs, the proportion of users was slightly higher among males than females, with benzodiazepines as the only exception. The differences observed between male and female illegal drug consumption were significant for all types of drugs except for opiates $(\mathrm{P}=0.28)$.

\section{Consumption of psychoactive substances before entry and during visit to the recreational site}

Table 3 describes the use of alcohol and illegal drugs reported by the participants prior to entering the club and during the evening of the intervention. Approximately half of the respondents $(47.1 \%$ of the males and $51.1 \%$ of the females) reported being completely sober before entering the club, whereas more than one-third of the participants $(40.7 \%$ of males and $36.0 \%$ of females) reported that they had consumed two or more drinks. The proportion of subjects that selfreported binge drinking (more than 4 drinks) prior to entering the recreational sites was $19.6 \%$ of the males and $12.8 \%$ of the females $(\mathrm{P}=0.001)$.

Table 1. Characteristics of the sample, including socio-demographic variables and psychoactive substance consumption.

\begin{tabular}{|c|c|c|c|}
\hline & $\begin{array}{c}\text { Male } \\
(\mathrm{N}=3188) \\
\%\end{array}$ & $\begin{array}{c}\text { Female } \\
(\mathrm{N}=1507) \\
\%\end{array}$ & $\mathbf{P}$ \\
\hline Age group (years) & & & 0.002 \\
\hline$-15-19$ & 22.9 & 24.2 & \\
\hline$-20-24$ & 45.5 & 49.6 & \\
\hline$-25-29$ & 21.8 & 18.7 & \\
\hline$-30-35$ & 9.8 & 7.5 & \\
\hline Living situation & & & $<0.001$ \\
\hline Alone & 18.9 & 11.8 & \\
\hline With parents & 63.0 & 62.4 & \\
\hline With partner & 10.0 & 14.2 & \\
\hline With friends/colleagues & 6.0 & 8.5 & \\
\hline Other & 2.1 & 3.1 & \\
\hline Education & & & $<0.001$ \\
\hline Academic & 25.8 & 36.6 & \\
\hline High school & 53.0 & 50.0 & \\
\hline Middle school & 17.4 & 10.3 & \\
\hline None & 3.8 & 3.1 & \\
\hline Employment & & & $<0.001$ \\
\hline Employed & 49.3 & 36.0 & \\
\hline Seeking employment & 7.6 & 7.3 & \\
\hline Student & 43.1 & 56.7 & \\
\hline Frequency of alcohol use in the past month & & $<0.001$ & \\
\hline Never & 7.0 & 10.5 & \\
\hline Once or twice per month & 18.6 & 30.3 & \\
\hline Once per week & 37.6 & 40.2 & \\
\hline 3 or 4 days per week & 25.2 & 15.7 & \\
\hline Daily & 11.6 & 3.3 & \\
\hline Drunkenness in the past month: yes & 56.5 & 45.0 & $<0.001$ \\
\hline Illegal drug consumption in the last year & & $<0.001$ & \\
\hline Never & 63.9 & 69.7 & \\
\hline Once & 6.8 & 5.4 & \\
\hline Sometimes & 15.3 & 15.7 & \\
\hline Several times per month & 4.3 & 2.8 & \\
\hline Several times per week & 9.7 & 6.4 & \\
\hline
\end{tabular}

We found that $79.8 \%$ of the males and $69.7 \%$ of the females reported alcohol consumption in the club, and $18.0 \%$ of the males and $11.0 \%$ of the females reported binge drinking $(\mathrm{P}<0.001)$.

With regard to the type of drink consumed during the night (data not shown), male drinkers reported consuming beer and distilled spirits (approximately $35 \%$ and $25 \%$, respectively), whereas females reported consuming alcoholpops (22.2\%), distilled spirits and beer (both 19.1\%).

Psychoactive drug use prior to entry was reported by $8.5 \%$ of the males and $6.0 \%$ of the females $(\mathrm{P}=0.002)$, and consumption of these drugs inside the recreational site was confirmed by $5.5 \%$ and $3.9 \%$ of the male and female respondents, respectively $(\mathrm{P}=0.059)$. The questionnaire also investigated the consumption of more than one illegal drug within the recreational site by self-reported psychoactive substance users (poly-drug consumption, alcohol excluded). Poly-drug use was reported by $71.2 \%$ of the male users and $66.6 \%$ of the female users $(\mathrm{P}=0.733)$.

\section{Multivariate analysis}

A multivariate analysis was performed to investigate the potential predictors of binge drinking. Socio-economic variables and illegal consumption of marijuana and cocaine were analysed in the univariate analysis to obtain crude ORs for inclusion in the multivariate logistic

Table 2. Illegal drug consumption in the last year by gender.

\begin{tabular}{lccc} 
& $\begin{array}{c}\text { Male } \\
(\mathrm{N}=3188) \\
\%\end{array}$ & $\begin{array}{c}\text { Female } \\
(\mathrm{N}=1507)\end{array}$ & P \\
& 31.9 & 26.9 & $<0.001$ \\
Marijuana & 5.3 & 3.7 & 0.021 \\
Methamphetamines & 4.0 & 2.5 & 0.009 \\
Hallucinogens & 4.5 & 2.9 & 0.009 \\
Amphetamines & 4.1 & 6.7 & $<0.001$ \\
Benzodiazepines & 1.6 & 1.2 & 0.28 \\
Opiates & 10.1 & 5.8 & $<0.001$ \\
\hline Cocaine & & &
\end{tabular}

Table 3. Consumption of alcohol and illegal drugs during the evening by gender.

\begin{tabular}{|c|c|c|c|}
\hline & $\begin{array}{c}\text { Male } \\
(\mathrm{N}=3188) \\
\%\end{array}$ & $\begin{array}{c}\text { Female } \\
(\mathrm{N}=1507) \\
\%\end{array}$ & $P$ \\
\hline \multicolumn{3}{|c|}{$\mathrm{N}^{\circ}$ of alcoholic drinks consumed prior to entry } & 0.001 \\
\hline 0 & 47.1 & 51.1 & \\
\hline 1 & 12.2 & 12.9 & \\
\hline 2 & 9.6 & 11.5 & \\
\hline 3 & 6.1 & 7.3 & \\
\hline 4 & 5.4 & 4.4 & \\
\hline$>4$ & 19.6 & 12.8 & \\
\hline \multicolumn{3}{|l|}{$\mathrm{N}^{\circ}$ of alcoholic drinks consumed at the club } & $<0.001$ \\
\hline 0 & 20.2 & 30.3 & \\
\hline 1 & 15.6 & 20.0 & \\
\hline 2 & 19.5 & 17.5 & \\
\hline 3 & 17.0 & 14.1 & \\
\hline 4 & 9.7 & 7.1 & \\
\hline$>4$ & 18.0 & 11.0 & \\
\hline Illegal drug consumption prior to entry & 8.5 & 6.0 & 0.002 \\
\hline Illegal drug consumption at the club & 5.5 & 3.9 & 0.059 \\
\hline \multicolumn{3}{|l|}{ Poly-drug consumption at the club* } & 0.733 \\
\hline Two substances & 63.2 & 57.1 & \\
\hline Three substances & 5.6 & 7.1 & \\
\hline More than three substances & 2.4 & 2.4 & \\
\hline
\end{tabular}

*Alcohol excluded. 
model to predict the probability of binge drinking. Table 4 shows the crude and adjusted ORs of the variables that remained statistically significant after the stepwise backward elimination analysis.

The univariate analysis found that 20- to 24-year-olds were the age group with the highest likelihood of binge drinking behaviour (crude OR 1.34, 95\% CI 1.08-1.66). However, when the model was adjusted for the main confounding factors, the association between age and binge drinking was attenuated (adjusted OR 1.14, $\mathrm{P}=0.3$ ). After controlling for the main confounding factors, living with a partner (adjusted OR $1.57,95 \%$ CI $1.13-2.19, \mathrm{P}=0.01$ ) and seeking employment (adjusted OR $1.66,95 \%$ CI $1.17-2.36, \mathrm{P}=0.005)$ were associated with a higher likelihood of alcohol abuse. The univariate analysis suggested a protective role for the highest educational levels, but after adjustment, only those who attended high school had a lower likelihood of alcohol abuse behaviour (adjusted OR 0.38, 95\% CI 0.25 - 0.58, $\mathrm{P}<0.001$ ).

An increased likelihood of binge drinking was evident in cannabis consumers, among whom the risk of binge drinking increased with the frequency of consumption (once, but not anymore: crude OR 1.52; $\mathrm{P}=0.01$; sometimes: crude $\mathrm{OR} 2.23, \mathrm{P}<0.001$; several times a month: crude OR 2.61, $\mathrm{P}<0.001$; several times a week: crude $0 \mathrm{R} 4.29 \mathrm{P}<0.001$ ), and among cocaine users (once but not anymore: crude OR 2.92 , $\mathrm{P}=0.01$; sometimes: crude $0 \mathrm{R} 2.98, \mathrm{P}<0.001$; several times a month: crude OR 4.18, $\mathrm{P}=0.004)$. The Brier score (the lower the score, the better the model performance) and the discrimination index c were 0.09 and 0.73 , respectively. Even after adjusting for age, education and socio-economic variables, the relationship between engaging in binge drinking and the frequency of marijuana use was apparent (once but not anymore: adjusted OR $1.45, \mathrm{P}=0.03$; sometimes: adjusted $\mathrm{OR} 1.96$, $\mathrm{P}<0.001$; several times a month: adjusted $\mathrm{OR} 1.94, \mathrm{P}=0.007$; several times a week: adjusted OR 3.66, $\mathrm{P}<0.001)$.

The nomogram of the logistic regression model is shown in Figure 1. Following the indications reported in the methods, it was easy to identify the profiles that were most at risk for binge drinking (for each variable). The characteristics most related to binge drinking were age 20 -

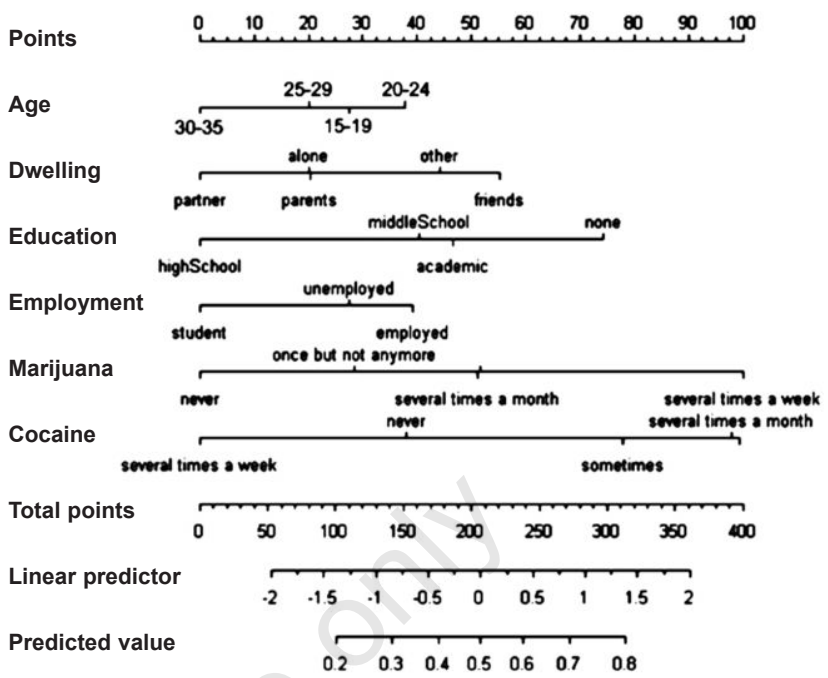

Figure 1. Nomogram for calculating the probability of binge drinking given an individual characteristic.

Table 4. Potential predictors of binge drinking.

\begin{tabular}{|c|c|c|c|c|c|c|}
\hline & Crude OR & $(95 \% \mathrm{CI})$ & P & Adjusted OR & $(95 \% \mathrm{CI})$ & $P$ \\
\hline \multicolumn{7}{|l|}{ Age group (years) } \\
\hline $15-19$ & -- & & & -- & & \\
\hline $20-24$ & 1.34 & $(1.08-1.66)$ & 0.01 & 1.14 & $(0.89-1.46)$ & 0.3 \\
\hline$-25-29$ & 1.09 & $(0.84-1.41)$ & 0.51 & 0.91 & $(0.65-1.27)$ & 0.6 \\
\hline$-30-35$ & 0.74 & $(0.52-1.03)$ & 0.07 & 0.70 & $(0.46-1.07)$ & 0.1 \\
\hline \multicolumn{7}{|l|}{ Living situation } \\
\hline With parents & -- & & & -- & & \\
\hline Alone & 1.01 & $(0.81-1.27)$ & 0.9 & 0.99 & $(0.78-1.27)$ & 0.97 \\
\hline With partner & 0.78 & $(0.61-1.01)$ & 0.06 & 1.57 & $(1.13-2.19)$ & 0.01 \\
\hline With friends & 1.81 & $(1.33-2.48)$ & $<0.001$ & 0.77 & $(0.57-1.03)$ & 0.08 \\
\hline Other & 1.32 & $(0.80-2.20)$ & 0.3 & 1.36 & $(0.79-2.34)$ & 0.3 \\
\hline \multicolumn{7}{|l|}{ Education } \\
\hline None & -- & & & -- & & \\
\hline Middle school & 0.69 & $(0.45-1.07)$ & 0.1 & 0.64 & $(0.41-1.02)$ & 0.06 \\
\hline High school & 0.43 & $(0.29-0.65)$ & $<0.001$ & 0.38 & $(0.25-0.58)$ & $<0.001$ \\
\hline Academic & 0.71 & $(0.47-1.07)$ & 0.1 & 0.70 & $(0.45-1.08)$ & 0.11 \\
\hline \multicolumn{7}{|l|}{ Employment } \\
\hline Student & -- & & & -- & & \\
\hline Seeking employment & 1.23 & $(0.88-1.71)$ & 0.2 & 1.66 & $(1.17-2.36)$ & 0.005 \\
\hline Employed & 1.57 & $(1.13-2.18)$ & 0.007 & 1.43 & $(1.00-2.04)$ & 0.05 \\
\hline \multicolumn{7}{|l|}{ Marijuana consumption } \\
\hline Never & -- & & & -- & & \\
\hline Once, but not anymore & 1.52 & $(1.09-2.13)$ & 0.01 & 1.45 & $(1.02-2.04)$ & 0.03 \\
\hline Sometimes & 2.23 & $(1.74-2.86)$ & $<0.001$ & 1.96 & $(1.51-2.54)$ & $<0.001$ \\
\hline Several times per month & 2.61 & $(1.69-4.04)$ & $<0.001$ & 1.94 & $(1.20-3.14)$ & 0.007 \\
\hline Several times per week & 4.29 & $(2.79-6.62)$ & $<0.001$ & 3.66 & $(2.27-5.90)$ & $<0.001$ \\
\hline \multicolumn{7}{|l|}{ Cocaine consumption } \\
\hline Never & -- & & & -- & & \\
\hline Once, but not anymore & 2.92 & $(1.74-4.92)$ & $<0.001$ & 2.22 & $(1.28-3.83)$ & 0.004 \\
\hline Sometimes & 2.98 & $(1.83-4.87)$ & $<0.001$ & 1.68 & $(0.99-2.85)$ & 0.06 \\
\hline Several times per month & 4.18 & $(1.56-11.17)$ & 0.004 & 2.17 & $(0.77-6.14)$ & 0.14 \\
\hline Several times per week & 1.04 & $(0.19-5.71)$ & 0.96 & 0.61 & $(0.10-3.88)$ & 0.6 \\
\hline
\end{tabular}


24 years, living with friends, lack of education, employment and frequent marijuana and cocaine use.

\section{Discussion}

This paper reports results from the TEN-D by Night survey, which aimed to investigate the patterns of psychoactive substance consumption and the prevalence of binge drinking and poly-drug use in a large sample of young Europeans. The limitations and strengths of our study have been extensively discussed in a previous paper. ${ }^{22,27}$ In brief, the TEN-D survey has some strengths compared with other surveys on the same topic. Most studies examining alcohol and illegal drug consumption among young people in the nightlife context have used only selfreported measures. ${ }^{20,21}$ In the limited number of studies in which an objective measure of alcohol level was obtained, samples sizes were small or originated from very specific settings. ${ }^{15-31}$ By contrast, our survey combined self-reported alcohol and illegal drug use with objective testing, thus reducing the potential for bias. Moreover, our sample was recruited at typical recreational sites, which were not considered settings for major risk (such as rave parties). The portal survey technique conducts a temporal evaluation of substance use and provides pre- and post-assessment of consumption, ensuring the most reliable measure of substance effects. ${ }^{15}$ This study also has some limitations. First, the study design is cross-sectional; therefore, we were able to document only the correlations between variables, not the presence of a causal relationship. Second, the voluntary recruitment of the participants and the opportunistic selection of the recreational sites may have introduced a selection bias, limiting the representativeness of the sample and the generalisation of the results. However, the guarantee of anonymity reduced the number of young people who refused to participate. The data on illegal drug use should be interpreted with extra caution because consumption may be underestimated due to underreporting. However, we evaluated this assumption using the saliva test, which provided the opportunity to estimate the number of false declarations in both contexts. ${ }^{27}$ Our findings confirm the previously reported pattern of alcohol consumption among young people, known as the weekend drinking culture, especially among those in the 20 - to 24 -yearold age group. ${ }^{6,32}$ Moreover, the results from our analysis confirm that females usually drink less than males, and a higher percentage of females reported being teetotalers. ${ }^{6,14,33}$ Interestingly, the portal survey technique showed that a very large proportion of subjects had already consumed alcohol prior to entering the recreational site. This finding suggests that alcohol misuse is not limited to clubs. The survey on alcohol consumption should be expanded to other settings. Our results also confirm that binge drinking is a common practice among young people who visit clubs. ${ }^{13-16}$ With regard to this issue, there were no significant differences between males and females. The TEN-D survey confirmed the narrowing of the gap between males and females in terms of unhealthy lifestyles. ${ }^{18,32,34-37}$ Furthermore, binge drinking appeared to be linked to other independent risk factors, including age (with 20-to 24-year-olds at highest risk), living situation (living with friends), education (none or low), and illegal drug consumption (marijuana and cocaine). This finding confirms the results of other studies analysing the growing phenomenon of concurrent alcohol and psychoactive substance use in young men and women. ${ }^{16,18,38-40}$ Other studies, primarily conducted in United States, have found that unhealthy lifestyles are associated with certain risk factors, including the influence of college students' distance from family, the level of education, and the protective role of marriage. ${ }^{1,33,35,38,40}$ We took these factors into account in our study. Although binge drinking and the correlation between alcohol consumption and illegal drug use have been described extensively in the literature, the contribution of our European survey is the use of data collected directly from a location in which psychoactive substances are usually consumed. Regarding illegal drug use, our study found that many youths reported having tried one or more substances in the last year, most frequently marijuana (one-third of the sample) and cocaine. These data are in agreement with those reported in the literature, but our study had a larger sample size than previous surveys $5^{5,6,9,20,21}$ because of the specific setting and timing of our study intervention, which was conducted at recreational locations on weekend nights. The illegal drug consumption at the recreational sites was lower than that reported by other surveys, especially for methamphetamines, hallucinogens and opiates. It is possible that the particular setting of these surveys, which were usually conducted in the context of rave parties, captured the behaviours of a different population at an increased risk of use. ${ }^{18,21}$ More than $70 \%$ of those who reported substance use during the visit to the club reported consuming more than one drug. These data confirm previous literature showing that a majority of substance users are also poly-drug users. . $^{18,38}$

\section{Conclusions}

The TEN-D by Night survey showed that it is possible to identify individuals and groups at high risk. This identification allows for a focus on specific targets and facilitates the reshaping of prevention programmes. The increasing use of psychoactive substances among younger generations should be studied extensively to promote successful intervention campaigns. These interventions should consider locations, in addition to discos and clubs, where young people consume alcohol and use illicit drugs.

\section{TEN-D Group}

B. Alamar, F. Alonso, C. Calatayud, F. Carena, S. Chiadò Piat, T. Chiapusso, CONSEPI group, A. Druart, M.T. Duce, C. Esteban, C. Izzicupo, Ag. Kalitowicz, An. Kalitowicz, M. Kedzia, G. Manassero, E. Pignata, R. Papalia, I. Pudule, L. Sànchez, R. Siliquini, V. Siliquini, R. Sroka, D. Vankov, S. Vanzino, A. Villerusa.

Correspondence: Dr. Roberta Siliquini, Department of Public Health, University of Torino, via Santena 5/bis, 10141 Torino, Italy.

Tel. +39.011.6705875 - Fax: +39.011 .6705889$

E-mail: roberta.siliquini@unito.it

Key words: young people, alcohol, substance abuse, binge drinking, lifestyle Acknowledgments: the authors gratefully acknowledge the young drivers who participated in this study and the students of the University of Torino for their help during the training session of the survey staff.

Funding: the TEN-D by Night study is supported by the Public Health Executive Agency - European Commission, Grant n. 2007326.

Contributions: RS, study design, paper revision; AC, manuscript drafting; PB, data analysis; FB, data analysis and paper revision; TEN-D Group, study conduction, data collecting.

Conflict of interest: the authors declare no conflicts of interest.

Received for publication: 18 November 2011.

Accepted for publication: 16 February 2012.

(C) Copyright R. Siliquini et al., 2012

Licensee PAGEPress, Italy

Journal of Public Health Research 2012; 1:e15

doi:10.4081/jphr.2012.e15

This work is licensed under a Creative Commons Attribution NonCommercial 3.0 License (CC BY-NC 3.0). 


\section{References}

1. Lange JE, Clapp JD, Turrisi R, et al. College binge drinking: what is it? Who does it? Alcohol Clin Exp Res 2002 May;26:723-30.

2. Berg HY. Reducing crashes and injuries among young drivers: what kind of prevention should we be focusing on? Inj Prev 2006;12:158.

3. Galanti MR, Siliquini R, Cuomo L, et al. Testing anonymous link procedures for follow-up of adolescents in a school-based link procedures for follow up of adolescents in a school-based trial: the EUDAP pilot study. Prev Med 2007;44:174-7.

4. Siliquini R, Faggiano F, Genianatti S, et al. Patterns of drugs use among young men in Piedmont (Italy). Drug Alcohol Depend 2001;64:329-35.

5. European Monitoring Centre for Drugs and DrugAddiction, EMCDDA. Annual Report on the State of the drugs problem in Europe. 2011. Accessed on: 6 December 2011. Available from: http:/www.emcdda.europa.eu/publications/annual-report/2011

6. European School Survey Project on Alcohol and other Drugs, ESPAD. The 2007 ESPAD Report. Accessed on: 6 December 2011. Available from: http://www.espad.org/documents/Espad/ ESPAD_reports/2007/The_2007_ESPAD_Report-FULL_091006.pdf

7. Rome ES. It's a rave new world: rave culture and illicit drug use in the young. Cleve Clin J Med 2001;68:541-50.

8. Mura P, Chatelain C, Dumestre V, et al. Use of drugs of abuse in less than 30-year-old drivers killed in a road crash in France: a spectacular increase for cannabis, cocaine and amphetamines. Forensic Sci Int 2006;160:168-72.

9. Hoare J. Nationally representative estimates of illicit drugs use by self-reported sexual orientation, 2007/08 and 2008/09 BCS. Accessed on: 6 December 2011. Available from: http:/www.homeoffice.gov.uk/publications/science-research-statistics/research-statistics/crime-research/hosb1310/hosb13101-annex2?view=Binary

10. Rupp TL, Acebo C, Seifer R, Carskadon MA. Effects of a moderate evening alcohol dose. II: performance. Alcohol Clin Exp Res 2007;31:1365-71.

11. National Highway Traffic Safety Administration, NHTSA. Traffic Basic Facts 2007. Accessed on: 2 August 2010. Available from: http://www-nrd.nhtsa.dot.gov/Pubs/810993.pdf

12. Lange JE, Lauer EM, Voas RB. A survey of the San Diego-Tijuana cross-border binging. Methods and analysis. Eval Rev 1999;23:37898.

13. Barquín J, Luna Jde D, Hernández AF. A controlled study of the time-course of breath alcohol concentration after moderate ingestion of ethanol following a social drinking session. Forensic Sci Int 2008;177:140-5.

14. Trerotoli P, Soldano S, Serio G, Moretti L. Drinking habits and performance in an attention test in young people frequenting discotheques. Ann Ig 2005; 17:47-55.

15. Voas RB, Furr-Holden D, Lauer E, et al. Portal surveys of time-out drinking locations: a tool for studying binge drinking and AOD use. Eval Rev 2006;30:44-65.

16. Courtney KE, Polich J. Binge drinking in young adults: data, definitions, and determinants. Psychol Bull 2009;135:142-56.

17. Farke W, Anderson P. Binge drinking in Europe. Adicciones 2007;19333-9.

18. Chinet L, Stéphan P, Zobel F, Halfon 0. Party drug use in techno nights: a field survey among French-speaking Swiss attendees. Pharmacol Biochem Behav 2007;86:284-9.

19. Furr-Holden D, Voas RB, Kelley-Baker T, Miller B. Drug and alcoholimpaired driving among electronic music dance event attendees. Drug Alcohol Depend 2006;85:83-6.
20. Parsons JT, Grov C, Kelly BC. Club drug use and dependence among young adults recruited through time-space sampling. Public Health Rep 2009;124:246-54.

21. Ramo DE, Grov C, Delucchi K, et al. Typology of club drug use among young adults recruited using time-space sampling. Drug Alcohol Depend 2010;107:119-27.

22. Siliquini R, Chiadò Piat S, Alonso F, et al. A European study on alcohol and drug use among young drivers: the TEND by Night study design and methodology. BMC Public Health 2010;10:205.

23. Schlesselman JJ. Case-control studies: design, conduct, analysis. 1982, Oxford University Press, New York, USA.

24. Drägenwerk AG \& Co. KGaA, 2010. Alcohol Test website. Accessed on: 6 December 2011. Available from: http:/www.drager.ru/ST/ internet/pdf/Master/En/gt/Alcodrug/9044871_Alcotest6510_e.pdf

25. Simucar Driving Simulator website. Accessed on: 2 August 2010. Available from URL: http://www.simucar.com/en/simulators/ SimuNomad_3ecrans.html

26. AlcolPro-Drug Test \& Alcohol Test Products, 2009. Oral fluid drug screen device website. Accessed on: 6 December 2011. Available from: http://www.alcopro.com/mm5/merchant.mvc?Screen= PROD\&Store_Code=A\&Product_Code $=823$ B \&Categorycode $=$ SALI VA-DRUG-TEST

27. Siliquini R, Bert F, Alonso F, et al. Correlation between drivingrelated skill and alcohol use in young-adults from six European countries: the TEN-D by Night Project. BMC Public Health 2011;11:526.

28. Harrell FE, Lee KL, Mark DB. Multivariable prognostic models: Issues in developing models, evaluating assumptions and adequacy, and measuring and reducing errors. Stat Med 1996;1549:361-87.

29. R Development Core Team. R: A language and environment for statistical computing. R Foundation for Statistical Computing. 2009, Vienna, Austria.

30. Harrell FE. Regression modeling strategies: with applications to linear models, logistic regression, and survival analysis. 2001, Springer, New York, USA.

31. Miller BA, Furr-Holden D, Johnson MB, et al. Biological markers of drug use in the club setting. J Stud Alcohol Drugs 2009;70:261-8.

32. Kuntsche E, Cooper ML. Drinking to have fun and to get drunk: motives as predictors of weekend drinking over and above usual drinking habits. Drug Alcohol Depend 2010;110:259-62.

33. Slutske WS. Alcohol use disorders among US college students and their non-college-attending peers. Arch Gen Psychiat 2005;62:3217.

34. Balodis IM, Potenza MN, Olmstead MC. Binge drinking in undergraduates: relationships with sex, drinking behaviors, impulsivity, and the perceive effects of alcohol. Behav Pharmacol 2009;20:51826.

35. Grucza RA, Norberg KE, Bierut LJ. Binge drinking among youths and young adults in the United States: 1979-2006. J Am Acad Child Psy 2009;48:692-702.

36. Miller J , Naimi T, Brewer R, Jones SE. Binge drinking and associated health risk behaviors among high school students. Pediatrics 2007;119:76-85.

37. Kelly-Weeder S. Binge drinking in college-aged women: framing a gender-specific prevention strategy. J Am Acad Nurse Pract 2008;20:577-84.

38. Grov C, Kelly BC, Parsons JT. Polydrug use among club-going young adults recruited through time-space sampling. Subst Use Misuse 2009;44:848-64.

39. Hughes E, Kilmer G, Li Y, et al. Surveillance for certain health behaviors among states and selected local areas - United States, 2008. MMWR Surveill Summ 2010;59:1-221.

40. Naimi TS, Nelson DE, Brewer RD. The intensity of binge alcohol consumption among U.S. adults. Am J Prev Med 2010;38:201-7. 